\title{
Benefit of FSH priming of women with PCOS to the in vitro maturation procedure and the outcome: a randomized prospective study
}

\author{
A. L. Mikkelsen and S. Lindenberg \\ The Fertility Clinic, Herlev University Hospital, Institute for Human Reproduction, \\ Fruebjergvej 3, DK-2100 Copenhagen, Denmark
}

\begin{abstract}
The aim of this study was to determine whether the rates of in vitro oocyte maturation, fertilization and cleavage, as well as implantation rate and pregnancy rate, could be improved by low-dose priming with FSH in vivo before retrieval of immature oocytes in patients with polycystic ovary syndrome (PCOS). From March 1998 to June 2000, a total of 28 women underwent 36 completed treatment cycles, randomized sequentially in one of two groups. Women in group 1 ( $n=12$ cycles) received no stimulation and women in group $2(n=24$ cycles) received 150 iu recombinant $\mathrm{FSH}$ day $^{-1}$ for 3 days, initiated on day 3 after menstruation. Aspiration was performed transvaginally between day 9 and day 17 in the unstimulated group and on day 8 or day 9 in the FSH-primed group after FSH deprivation for 2 or 3 days. All cumulus-enclosed oocytes of healthy appearance were matured in culture medium (TCM-199) in vitro for 28-36 h before intracytoplasmic sperm injection (ICSI). After oocyte retrieval the women were given
\end{abstract}

oestradiol (6 mg day ${ }^{-1}$ ) and progesterone administration (300 $\mathrm{mg} \mathrm{day}^{-1}$ ) was initiated 2 days later. Suitable embryos (maximum two embryos) were transferred on day 3 after ICSI. The percentage of oocytes reaching metaphase II was significantly higher $(P<0.05)$ in the $\mathrm{FSH}$-primed group $(59 \%, 92 / 156)$ compared with the non-primed group $(44 \%$, $36 / 81)$. There were no significant differences in the rates of oocyte fertilization and cleavage between these groups. No pregnancies were obtained in group $1(0 \%, 0 / 12)$, whereas seven clinical pregnancies were obtained in group $2(29 \%$, $7 / 24)(P<0.05)$. In group 2,37 embryo transfers resulted in eight implantations $(21.6 \%)$. Three healthy singleton children have been born at term; the remaining pregnancies ended with spontaneous abortions in the first trimester. These results indicate that priming with recombinant FSH before harvesting of immature oocytes from patients with PCOS may improve the maturational potential of the oocytes and the implantation rate of the cleaved embryos.

\section{Introduction}

Women with polycystic ovary syndrome (PCOS) are extremely sensitive to stimulation with exogenous gonadotrophins in assisted reproduction, and have a significant risk of developing ovarian hyperstimulation syndrome (OHSS). Different strategies for prevention of OHSS have been reported, including cancellation of the cycle, i.v. administration of albumin (Shalev et al., 1995), laparoscopic ovarian electrocautery before gonadotrophin therapy (Rimington et al., 1997), total embryo cryopreservation with delayed uterine transfer (Tittinen et al., 1995), and 'coasting', including the withholding of hCG administration (Sher et al., 1995). However, at present there is no method that provides absolute prevention or prediction of OHSS.

It has been postulated that immature oocyte retrieval combined with in vitro maturation (IVM) could potentially replace standard stimulated in vitro fertilization (IVF) in women with PCOS in the future. This strategy would eliminate the risk of hyperstimulation syndrome and reduce the cost and duration of treatment. Several studies report the use of in vitro

Email: alm@ciconia.dk matured and fertilized oocytes in women with PCOS (Cha and Chian, 1998; Trounson et al., 1998). Although immature oocytes retrieved from untreated ovaries can be matured, fertilized and developed in vitro, the rate of implantation of the cleaved embryos is low (Cha et al., 2000). The maturation rate of immature oocytes retrieved from patients with PCOS is impaired compared with that in women undergoing regular cycles (Barnes et al., 1996). Priming with FSH (Suikkari et al., 2000) or hCG (Chian et al., 2000) has been suggested before oocyte pick-up to compensate for this impairment. The aim of the present study was to determine whether priming with $\mathrm{FSH}$ before immature oocyte retrieval alters the rates of oocyte maturation, fertilization, cleavage, implantation and pregnancy in women with PCOS.

\section{Materials and Methods}

\section{Patients}

The study included 28 couples with infertility of at least 1 year in duration. The couples were referred for IVF-ICSI due to tubal damage, male factor infertility or previous unsuccessful ovarian stimulation cycles with up to three 
previous unsuccessful IVF-ICSI treatments. All women had an ultrasonographic diagnosis of PCOS, defined as $>10$ follicles $2-8 \mathrm{~mm}$ in diameter, in one plane located either around or through an echodense thickened stroma (Adams et al., 1985), and had ovulatory disorders with oligomenorrhoea or amenorrhoea for $>6$ months. The study was open to patients up to the age of 38 years whose body mass index was between $18 \mathrm{~kg} \mathrm{~m}^{-2}$ and $29 \mathrm{~kg} \mathrm{~m}^{-2}$. All women were normoprolactinaemic.

The study was conducted from March 1998 to June 2000 in a total of 36 completed treatment cycles. The patients were assigned randomly to two groups. Women in group 1 ( $n=12$ cycles) received no stimulation, whereas women in group 2 ( $n=24$ cycles) received 150 iu recombinant FSH day $^{-1}$ (Gonal-F; Serono, Genova) for 3 days, initiated on day 3 after menstruation.

The local ethics committee approved the study. All couples participated in the study after giving their informed written consent.

The patients were examined on day 3 after spontaneous menses for those patients who reported some menstrual function, or on day 3 after withdrawal bleeding induced by $10 \mathrm{mg}$ medroxyprogesterone (Perlutex; Leo, Ballerup) administered orally once a day. Five cycles were excluded because an ovarian cyst was detected by ultrasonography on day 3 . The remaining 36 cycles were randomized according to the ratio 1:2 (non-primed:primed). For each cycle, the number of oocytes, and the rates of maturation, fertilization, cleavage and pregnancy were calculated. In the non-primed group, aspiration was performed between day 9 and day 17, when the thickness of the endometrium was at least $5 \mathrm{~mm}$. Aspiration was performed in the $\mathrm{FSH}$ primed group on day 8 or day 9 after a delay of 2-3 days after the last injection of recombinant FSH.

\section{Oocyte recovery, maturation and fertilization, and embryo culture}

All procedures were performed as described by Smith et al. (2000). In brief, oocyte retrieval was performed transvaginally with a single lumen 17-G needle $250 \mathrm{~mm}$ in length (SwemedLab, Billdal) connected to a syringe to induce the aspiration pressure. During this procedure the women received a mild i.v. sedation with triazolam (Halcion; Pharmacia and Upjohn, Copenhagen) and alfentamil (Rapifen; Janssen-Cilag, Copenhagen). A paracervical block was placed at 2 and 10 o'clock 2-3 min before puncture through the vagina. The follicles were punctured, not flushed. After aspiration the needle was flushed with prewarmed Ham's F-10 medium with heparin (Life Technologies, Copenhagen). Follicular aspirates were filtered (Falcon 1060; $70 \mu \mathrm{m}$ mesh size) to remove erythrocytes and small cellular debris.

Oocytes with signs of atresia (dark or shrunken, irregular cytoplasm) or mechanical damage were discarded. Healthy cumulus-enclosed oocytes were matured for 28-36 h in TCM-199 (Sigma, Roedovre) to obtain metaphase II oocytes. The culture medium was supplemented with $0.3 \mathrm{mmol}$ sodium pyruvate $\mathrm{I}^{-1}, 1500$ iu penicillin $\mathrm{G} \mathrm{ml}^{-1}$, $50 \mathrm{mg}$ streptomycin sulphate $\mathrm{ml}^{-1}, 1 \mu \mathrm{g}$ oestradiol $\mathrm{ml}^{-1}$ (all from Sigma), 0.075 iu recombinant $\mathrm{FSH} \mathrm{ml} \mathrm{m}^{-1}$ (Gonal-F; Serono), 0.5 iu hCG ml-1 (Profasi; Serono) and heatinactivated serum from the patient (10\%). Oocytes were cultured singly in $25 \mu \mathrm{l}$ droplets of IVM medium under paraffin oil at $37^{\circ} \mathrm{C}$ in $5 \% \mathrm{CO}_{2}$ and humidified air for 28-36 h.

The oocytes were inseminated by ICSI, which enabled us to confirm the presence of the first polar body microscopically, which was the criterion used to classify oocytes as matured to metaphase II. The ICSI technique was similar to that described by Smith et al. (2000) and the injected oocytes were cultured individually in droplets of IVF medium (Medi-Cult, Copenhagen) in Falcon Petri dishes (Life Technologies) under oil at $37^{\circ} \mathrm{C}$ in $5 \% \mathrm{CO}_{2}$ and humidified air. Embryos were cultured to day 2.5 or 3.0 (day $0=$ day of insemination), at which time suitable embryos (maximum two embryos) were transferred into the uterus of the patient.

Endometrial priming consisted of oestradiol (2 mg administered orally three times a day; oestradiol 'DAK'; Nycomed, Roskilde), started on the day of oocyte retrieval. Two days after aspiration, treatment with intravaginal progesterone suppositories $(100 \mathrm{mg}$ three times a day; Progestan; Organon, Oss) was initiated. Administration of oestradiol and progesterone was continued until a pregnancy test was performed and was continued until day 50 of gestation if the pregnancy test was positive.

\section{Ultrasound examination}

Ultrasound examination was performed on day 3, days 6 or 7 and either daily or every second day until the day of aspiration using a 7.5 MHz transvaginal transducer (B \& $\mathrm{K}$ Medical, Gentofte). The follicular diameter was calculated as the mean of the longest follicular axis and the axis perpendicular to it. A clinical pregnancy was defined as ultrasonographic evidence of intrauterine fetal heart activity at week 5 after embryo transfer.

\section{Hormone assays}

The serum concentrations of $\mathrm{LH}, \mathrm{FSH}$, oestradiol, inhibin $A$ and inhibin B were determined on day 3, days 6 or 7 and the day of aspiration and evaluated retrospectively. Immunoreactive $\mathrm{FSH}$ concentrations were determined using an autoanalyser (Immuno I; Bayer, Copenhagen). The interassay coefficients of variation were $3.0 \%$ for values $<5.5$ iu $\mathrm{ml}^{-1}$ and $3.2 \%$ for values $<15$ iu $\mathrm{ml}^{-1}$. Oestradiol concentrations were measured using the same autoanalyser and interassay coefficients of variation were $10 \%$ for values $<0.1 \mathrm{nmol} \mathrm{I}^{-1}, 8 \%$ for values between $0.2 \mathrm{nmol} \mathrm{I}^{-1}$ and 1.0 $\mathrm{nmol} \mathrm{I}^{-1}, 6 \%$ for values between $1.0 \mathrm{nmol} \mathrm{I}^{-1}$ and $2.0 \mathrm{nmol}$ $\mathrm{I}^{-1}$ and $5 \%$ for values $>2 \mathrm{nmol} \mathrm{I}^{-1}$. Inhibin $A$ and inhibin $B$ concentrations were measured using specific ELISA kits (Serotec Ltd, Oxford). The assays are based on previously 
Table 1. Clinical variables of patients in unstimulated and FSH-primed groups

\begin{tabular}{lccccc}
\hline & Number & $\begin{array}{c}\text { Number } \\
\text { of cycles }\end{array}$ & $\begin{array}{c}\text { Number with male } \\
\text { factor infertility } \\
\text { and PCOS }\end{array}$ & $\begin{array}{c}\text { Number with tubal } \\
\text { factor infertility } \\
\text { and PCOS }\end{array}$ & $\begin{array}{c}\text { Median age } \\
\text { in years } \\
\text { (range) }\end{array}$ \\
\hline Group 1 (unstimulated) & 12 & 9 & 3 & 5 & $31(20-35)$ \\
Group 2 (FSH-primed) & 24 & 20 & 7 & 3 & $31(27-37)$ \\
\hline
\end{tabular}

PCOS: polycystic ovary syndrome.

Table 2. Number of oocytes obtained for in vitro maturation and rates of maturation, fertilization, cleavage and pregnancy in women in unstimulated and FSH-primed groups

\begin{tabular}{|c|c|c|c|c|c|c|c|}
\hline & $\begin{array}{l}\text { Number } \\
\text { of cycles }\end{array}$ & $\begin{array}{c}\text { Oocytes } \\
\text { for IVM }(\%)\end{array}$ & $\begin{array}{c}\text { Metaphase II } \\
(\%)\end{array}$ & $\begin{array}{l}\text { Two pronuclei } \\
(\% \text { MII })\end{array}$ & $\begin{array}{l}\text { Cleavage } \\
(\% \text { MII })\end{array}$ & $\begin{array}{c}\text { Pregnancy } \\
\text { rate/aspiration } \\
(\%)\end{array}$ & $\begin{array}{c}\text { Implantation } \\
(\%)\end{array}$ \\
\hline Group 1 (unstimulated) & 12 & $81(70)$ & $36(44)$ & $25(69)$ & $23(64)$ & 0 & $0 / 16(0)$ \\
\hline Group 2 (FSH-primed) & 24 & $156(73)$ & $92(59) *$ & $64(70)$ & $52(56)$ & $7 / 24(29)^{*}$ & $8 / 37(21.6)^{*}$ \\
\hline
\end{tabular}

IVM: in vitro maturation; MII: metaphase II

*Indicates significant difference compared with corresponding group 1 value $(P<0.05)$.

published methods for measuring inhibin A (Groome et al., 1994) and inhibin B (Groome et al., 1996). The detection limit of inhibin A was $7 \mathrm{pg} \mathrm{ml}^{-1}$ and the interassay coefficients of variation were $19.0 \%$ for values $<14.9 \mathrm{pg}$ $\mathrm{ml}^{-1}$ and $13.9 \%$ for values $<31 \mathrm{pg} \mathrm{ml}^{-1}$. The detection limit for inhibin B was $20 \mathrm{pg} \mathrm{ml}^{-1}$ and the interassay coefficients of variation were $19.0 \%$ for values $<46 \mathrm{pg} \mathrm{ml}^{-1}, 7 \%$ for values between $46 \mathrm{pg} \mathrm{ml}^{-1}$ and $238 \mathrm{pg} \mathrm{ml}^{-1}$ and $6.5 \%$ for values between $238 \mathrm{pg} \mathrm{ml}^{-1}$ and $634 \mathrm{pg} \mathrm{ml}^{-1}$.

\section{Statistical analyses}

Hormone concentrations are presented as median and range, as the data were not normally distributed, leading to the use of non-parametric tests. Mann-Whitney $U$ test was used to analyse differences between hormone variables. Either Fisher's exact test or chi-squared test was used to compare qualitative data. Values were considered significant at $P<0.05$.

\section{Results}

The ages of the women and indications for IVM are shown (Table 1).

A total of 115 oocytes was recovered in group 1 (nonstimulated group) and, of these, 81 (70\%) were cumulusenclosed and used in the experiment (median: 7 (range 2-12) oocytes per aspiration; Table 2). A total of 213 oocytes was recovered in group 2 (FSH primed group) and, of these, 156 (73\%) were cumulus-enclosed and used in the experiment (median: 7 (range 2-16) oocytes per aspiration; Table 2).

The percentage of oocytes reaching metaphase II was higher in the FSH-primed group $(59 \%, 92 / 156)$ than in the non-primed group $(44 \%, 36 / 81)(P<0.05)$. Fertilization and cleavage rates were similar between the groups (Table 2).

Three patients in group1 did not undergo embryo transfer because of fertilization failure ( $n=1$ cycle) or poor embryo development ( $n=2$ cycles). In total, 16 embryos were transferred into nine patients. No pregnancies were obtained and no embryos were cryopreserved. Three patients in group 2 did not undergo embryo transfer because of fertilization failure ( $n=1$ cycle) or poor embryo development ( $n=2$ cycles). In total, 37 embryos were transferred into 21 patients. Six singleton and one twin gestation with live fetuses were obtained, giving a pregnancy rate per aspiration of $29 \%$ (7/24), a pregnancy rate per transfer of $33 \%(7 / 21)$ and an implantation rate of $21.6 \%$ (8/37). A twin pregnancy reduced spontaneously to a singleton. Three healthy children have been born at term; the remaining pregnancies ended with spontaneous abortions in the first trimester. Cryopreservation and subsequent embryo transfer were performed for two patients (three embryos and one embryo, respectively). None of these resulted in pregnancy after thawing and transfer.

In the $\mathrm{FSH}$-primed group the median maximum diameter of the largest follicles on the day of aspiration was $11 \mathrm{~mm}$ (range 9-14 mm) and the remaining follicles were 5-8 mm in diameter. In the unprimed group, the size of the follicles was $<10 \mathrm{~mm}$ (median $6 \mathrm{~mm}$; range 5-9 mm). The rate of oocyte retrieval of the follicles was estimated to be $50 \%$.

The thickness of the endometrium on the day of aspiration did not differ between the two groups. Both groups had similar basal (day 3) hormone concentrations, except for the concentration of inhibin B, which was lower in the FSH-primed group (Table 3). After FSH priming, a significant increase in serum concentrations of oestradiol, inhibin A and inhibin B was observed from day 3 to days 6 
Table 3. Serum concentrations of $\mathrm{FSH}, \mathrm{LH}$, oestradiol, inhibin B and inhibin A on day 3, on days 6-7 and on the day of aspiration in unstimulated and $\mathrm{FSH}$-primed groups

\begin{tabular}{|c|c|c|c|c|c|c|}
\hline & \multicolumn{2}{|c|}{ Day 3 (range) } & \multicolumn{2}{|c|}{ Days 6-7 (range) } & \multicolumn{2}{|c|}{ Day of aspiration (range) } \\
\hline & Group 1 & Group 2 & Group 1 & Group 2 & Group 1 & Group 2 \\
\hline $\mathrm{FSH}\left(\mathrm{iu} \mathrm{l}^{-1}\right)$ & $\begin{array}{c}5.7 \\
(4.8-9.4)\end{array}$ & $\begin{array}{c}5.9 \\
(4.3-9.5)\end{array}$ & $\begin{array}{c}6.0 \\
(4.8-11.8)\end{array}$ & $\begin{array}{c}6.8 \\
(4.0-12.5)\end{array}$ & $\begin{array}{c}6.0 \\
(4.3-11.5)\end{array}$ & $\begin{array}{c}4.5 \\
(2.4-9.7)\end{array}$ \\
\hline LH (iu I-1) & $\begin{array}{c}7.65 \\
(4.3-14.0)\end{array}$ & $\begin{array}{c}7.5 \\
(4.3-22.3)\end{array}$ & $\begin{array}{c}9.6 \\
(6.1-18.5)\end{array}$ & $\begin{array}{c}3.1^{*} \\
(0.8-7.8)\end{array}$ & $\begin{array}{c}9.9 \\
(4.5-13.8)\end{array}$ & $\begin{array}{c}7.7 \\
(2.8-5.5)\end{array}$ \\
\hline Oestradiol (pmol I-1) & $\begin{array}{c}110 \\
(50-200)\end{array}$ & $\begin{array}{c}100 \\
(70-420)\end{array}$ & $\begin{array}{c}250 \\
(110-580)\end{array}$ & $\begin{array}{c}835^{*} \\
(70-3990)\end{array}$ & $\begin{array}{c}245 \\
(110-580)\end{array}$ & $\begin{array}{c}160 \\
(70-1990)\end{array}$ \\
\hline Inhibin B (pg ml-1) & $\begin{array}{c}172 \\
(122-294)\end{array}$ & $\begin{array}{c}137^{*} \\
(79-245)\end{array}$ & $\begin{array}{c}159 \\
(121-230)\end{array}$ & $\begin{array}{c}521^{*} \\
(33-4519)\end{array}$ & $\begin{array}{c}171 \\
(85-220)\end{array}$ & $\begin{array}{c}150 \\
(78-846)\end{array}$ \\
\hline Inhibin A (pg ml-1) & $\begin{array}{c}<7 \\
(<7-11)\end{array}$ & $\begin{array}{c}<7 \\
(<7-13)\end{array}$ & $\begin{array}{c}9 \\
(<7-14)\end{array}$ & $\begin{array}{c}47^{*} \\
(<7-319)^{*}\end{array}$ & $\begin{array}{c}11 \\
(<7-20)\end{array}$ & $\begin{array}{c}10 \\
(<7-185)\end{array}$ \\
\hline
\end{tabular}

Values are medians.

Group 1: unstimulated; group 2: FSH-primed.

*Indicates significant difference compared with corresponding group 1 value $(P<0.05)$.

or 7. After discontinuing FSH administration the concentrations of oestradiol, inhibin A and inhibin B decreased in all cycles except for one, and the concentrations on the day of aspiration did not differ from the basal concentrations. In contrast, LH concentrations decreased during stimulation and increased from days 6 or 7 to the day of aspiration.

Patients without $\mathrm{FSH}$ priming showed a slight but significant increase in the concentrations of oestradiol on days 6 or 7 compared with the basal concentrations, and no decrease was observed from days 6 or 7 to the day of aspiration. No differences in the concentrations of inhibin $\mathrm{B}$, inhibin $\mathrm{A}$ or $\mathrm{LH}$ were observed in this group over the study period.

No difference in the concentration of $\mathrm{FSH}$ was demonstrated over the study period in the two groups. On days 6 or 7 the concentrations of oestradiol, inhibin $A$ and inhibin B were significantly higher in the FSH-primed group than in the non-primed group, whereas the concentrations of $\mathrm{LH}$ were significantly lower in the $\mathrm{FSH}$-primed group. No differences in the concentrations of these hormones were observed between the groups on the day of aspiration.

\section{Discussion}

The number of oocytes aspirated from patients with PCOS in the present study (median 7.5 oocytes per aspiration) was higher than the number of oocytes obtained in a previous study including women undergoing regular menstruation with normal ovaries (median 4 oocytes per aspiration) (Mikkelsen et al., 2000). This finding is in accordance with previous observations (Barnes et al., 1996; Trounson et al., 1998; Cha and Chian, 1998). Suikkari et al. (2000) reported that a small dose of $\mathrm{FSH}$ in women undergoing regular menstruation might increase the yield of immature oocytes collected. In the present study, FSH priming did not increase the number of oocytes retrieved from patients with PCOS.
Trounson et al. (1994) described the first pregnancy and delivery of a healthy baby after IVM of immature oocytes obtained from a patient with PCOS. Recently, Cha et al. (2000) reported pregnancy rates of $27 \%$. This high pregnancy rate required multiple embryo transfer (mean 6.3 embryos transferred per patient) and a low (6.9\%) implantation rate was achieved. Barnes et al. (1996) compared rates of maturation, fertilization and cleavage between untreated regularly ovulating women and irregular or anovulatory polycystic women. In almost all the parameters analysed, oocytes from patients undergoing regular cycles performed better. The reason for this was not determined. To compensate for this, Trounson et al. (1998) and Suikkari et al. (2000) suggested priming with FSH before oocyte collection. Suikkari et al. (2000) showed that starting low-dose FSH priming in the luteal phase resulted in maturation and fertilization rates in women with PCOS comparable with those of regular cycling women. In contrast, Trounson et al. (1998) did not find any effect of a short FSH stimulation in patients with PCOS undergoing regular cycles. In the present study, an improved maturation rate to metaphase II was observed in vitro after FSH priming in vivo. This finding is consistent with a previous observation, in which germinal vesicle breakdown (GVBD) was observed after $30 \mathrm{~h}$ of in vitro culture when oocytes were obtained from stimulated cycles, compared with $48 \mathrm{~h}$ when oocytes were obtained from unstimulated cycles (Cha and Chian, 1998). In the present study, although there was no significant difference between the groups with respect to fertilization and cleavage, the pregnancy rate was significantly higher in group 2 compared with group 1 . The two groups did not differ with respect to clinical characteristics and, therefore, the observed differences in rates of maturation and pregnancy may be due to priming with $\mathrm{FSH}$ before aspiration.

Comparing our results with those of others we found that 
the maturation rate of unstimulated oocytes in our IVM system was somewhat lower than that reported previously, whereas the maturation rate in the FSH-primed group was comparable with previous reports (Trounson et al., 1994; Barnes et al., 1996; Cha et al., 2000; Chian et al., 2000; Suikkari et al. 2000). The pregnancy rate of $29 \%$ per aspiration in the FSH-primed group was comparable with the results of Cha et al. (2000), although the implantation rate $(21.6 \%)$ was higher in the present study. The spontaneous abortion rate in the present study (four of seven) is higher than the rate observed in a previous study of women with regular cycles undergoing IVM (two of 11) (Mikkelsen et al., 2000). A higher rate of spontaneous abortions has been described previously in PCOS patients compared with women undergoing regular cycles. The high rate of spontaneous abortion has not been solved by this method.

It is unclear precisely how FSH priming works. The granulosa cells of women with PCOS may possess intact aromatase activity and respond to exogenous $\mathrm{FSH}$ stimulation with the production of oestradiol in vivo and in vitro (Almahbobi et al., 1996). In the present study, the serum concentrations of oestradiol on days 6-7 were significantly higher in the FSH-primed group compared with the unprimed group. Granulosa cells from women with PCOS respond excessively to administered gonadotrophins with a subsequent increase in the serum concentrations of oestradiol, inhibin A and inhibin B (Anderson et al., 1998; Enskog et al., 2000). The observations in the present study are in accordance with these reports. When FSH administration was discontinued, a decrease in the concentrations of oestradiol, inhibin A and inhibin B was observed, as has been reported previously by Welt et al. (1999).

Not only $\mathrm{FSH}$ priming but also the following $\mathrm{FSH}$ deprivation caused by withholding exogenous $\mathrm{FSH}$ would be expected to enhance the competence of the oocytes. It has been hypothesized that oocyte differentiation may be incomplete during follicular growth and that oocytes from plateau phase follicles have increased competence. Studies in cattle indicate an advantage of using moderate follicle stimulation followed by a period of $\mathrm{FSH}$ deprivation to obtain optimal embryo production from bovine oocytes (Sirard et al., 1999). Similar studies in humans are lacking, but in a pilot study better results were obtained when in vivo priming was combined with a $72 \mathrm{~h}$ delay compared with a $24 \mathrm{~h}$ delay (Mikkelsen et al., 1998). Furthermore, it is known from patients undergoing 'coasting' during ovarian hyperstimulation that a coasting period of $\leqslant 4$ days does not compromise the cycle outcome, whereas a coasting period $>4$ days may be suboptimal for the oocytes and the cycle outcome (Waldenström et al., 1999). On the basis of these results the oocytes were collected 2-3 days after the last injection of $\mathrm{FSH}$ in the present study.

FSH priming resulted in an increased size of the follicles in the stimulated group compared with the unstimulated group. Durinzi et al. (1995) demonstrated that human oocytes appear to have a size-dependent ability to resume meiosis and complete maturation. Tsuji et al. (1985) reported that oocytes retrieved from follicles 9-15 $\mathrm{mm}$ in diameter completed meiotic maturation to metaphase II at a higher rate than did oocytes from follicles $3-4 \mathrm{~mm}$ in diameter. The observations in the present study are in accordance with these earlier reports, as an increased size of follicle corresponded to an increased maturation rate in the stimulated group.

In conclusion, the results of the present study indicate that priming with recombinant $\mathrm{FSH}$ before harvesting of immature oocytes from patients with PCOS may improve their potential to mature and develop into viable embryos.

This study was supported in part by Medi-Cult A/S.

\section{References}

Adams J, Franks S, Polson DW et al. (1985) Multifollicular ovaries: clinical and endocrine features and response to pulsative gonadotropinreleasing hormone Lancet 2 1375-1379

Almahbobi G, Andereriesz C, Hutchingson P, McFarlane JR, Wood C and Trounson A (1996) Functional integrity of granulosa cells from polycystic ovaries Clinical Endocrinology 44 571-580

Anderson RA, Groome NP and Baird DT (1998) Inhibin A and inhibin B in women with polycystic ovarian syndrome during treatment with $\mathrm{FSH}$ to induce mono-ovulation Clinical Endocrinology 48 577-584

Barnes FL, Kausche AK, Tiglias J et al. (1996) Production of embryos from in vitro-matured primary oocytes Fertility and Sterility 65 1151-1156

Cha KY and Chian RC (1998) Maturation in vitro of immature human oocytes for clinical use Human Reproduction Update 4 103-120

Cha KY, Han SY, Chung HM, Choi DH, Lim JM, Lee WS, Ko JJ and Yoon TK (2000) Pregnancies and deliveries after in vitro maturation culture followed by in vitro fertilisation and embryo transfer without stimulation in women with polycystic ovary syndrome Fertility and Sterility $\mathbf{7 3}$ 978-983

Chian RC, Buckett WM, Tulandi T and Tan SL (2000) Prospective randomized study of human chorionic gonadotrophin priming before immature oocyte retrieval from unstimulated women with polycystic ovarian syndrome Human Reproduction 15 165-170

Durinzi KL, Saniga EM and Lanzendorf SE (1995) The relationship between size and maturation in-vitro in the unstimulated human oocyte Fertility and Sterility 63 404-406

Enskog A, Nilsson L and Brännström M (2000) Peripheral blood concentrations of inhibin B are elevated during gonadotrophin stimulation in patients who later develop ovarian OHSS and inhibin A concentrations are elevated after OHSS onset Human Reproduction 15 $532-538$

Groome NP, Illingworth PJ, O'Brien M, Cooke I, Ganesan TS, Baird DT and McNeilly AS (1994) Detection of dimeric inhibin throughout the human menstrual cycle Clinical Endocrinology 40 717-723

Groome NP, Illingworth PJ, O'Brien M, Pai R, Rodger FE, Mather JP and McNeilly AS (1996) Measurement of dimeric inhibin B throughout the human menstrual cycle Journal of Clinical Endocrinology and Metabolism 81 1401-1405

Mikkelsen AL, Smith SD and Lindenberg S (1998) In-vitro maturation of immature human oocytes Human Reproduction 13 (Abstract Book 1) O-48

Mikkelsen AL, Smith S and Lindenberg S (2000) Impact of oestradiol and inhibin A concentrations on pregnancy rate in in vitro maturation Human Reproduction 15 1685-1690

Rimington MR, Walker SM and Shaw RW (1997) The use of laparoscopic ovarian electrocautery in preventing cancellation of in-vitro fertilization treatment cycles due to the risk of hyperstimulation syndrome in women with polycystic ovaries Human Reproduction 12 1443-1447 
Shalev E, Gilai Y, Matinsky M and Ben-Ami M (1995) Decreased incidence of severe ovarian hyperstimulation syndrome in high risk in-vitro fertilization patients receiving intravenous albumin: a prospective study Human Reproduction 10 1373-1376

Sher G, Zouves C, Feinman M and Maassarani G (1995) 'Prolonged coasting': an effective method for preventing severe ovarian hyperstimulation syndrome in patients undergoing in-vitro fertilization Human Reproduction $103107-3109$

Sirard MA, Picard L, Dery M, Coenen K and Blondin P (1999) The time interval between $\mathrm{FSH}$ administration and ovarian aspiration influences the development of cattle oocytes Theriogenology 51 699-708

Smith SD, Mikkelsen AL and Lindenberg S (2000) Development of human oocytes matured in vitro for 28 or 36 hours Fertility and Sterility 73 541-544

Suikkari A-M, Tulppala M, Tuuri T, Hovatta O and Barnes F (2000) Luteal phase start of low-dose FSH priming of follicles results in an efficient recovery, maturation and fertilisation of immature human oocytes Human Reproduction 15 747-751

Tittinen A, Husa LM, Tulppala M et al. (1995) The effect of cryopreservation in prevention of ovarian hyperstimulation syndrome British Journal of Obstetrics and Gynaecology 102 326-329
Trounson A, Wood C and Kausche A (1994) In vitro maturation and the fertilisation and developmental competence of oocytes recovered from untreated polycystic ovarian patients Fertility and Sterility 62 353-362

Trounson A, Anderiesz C, Jones GM, Kausche A, Lolatgis N and Wood C (1998) Oocyte maturation Human Reproduction 13 (Supplement 3) 52-62

Tsuji K, Sowa M and Nakano R (1985) Relationship between human oocyte maturation and different follicular sizes Biology of Reproduction 32 413-417

Waldenström U, Kahn J, Marsk L and Nilsson S (1999) High pregnancy rates and successful prevention of severe ovarian hyperstimulation syndrome by 'prolonged coasting' of very hyperstimulated patients: a multicentre study Human Reproduction 14 294-297

Welt CK, Adams JM, Sluss PM and Hall JE (1999) Inhibin A and inhibin B responses to gonadotropin withdrawal depends on stage of follicle development Journal of Clinical Endocrinology and Metabolism 84 2163-2169

Received 9 March 2001.

First decision 4 May 2001.

Accepted 12 June 2001. 